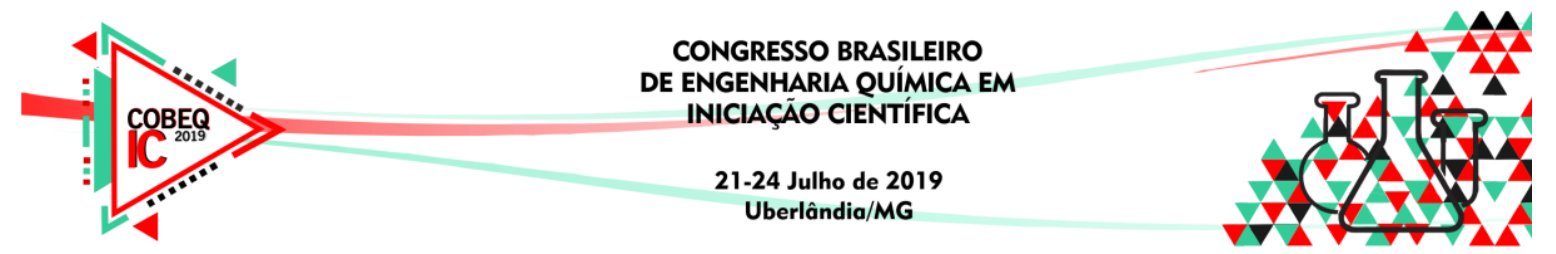

\title{
MODELAGEM E OTIMIZAÇÃO DOS PROCESSOS DE PIRÓLISE RÁPIDA E SUPER-RÁPIDA DE MADEIRA DE CARVALHO
}

\author{
I. B. CORREA ${ }^{1}$, L. S. SANTOS ${ }^{1}$ \\ ${ }^{1}$ Universidade Federal Fluminense, Departamento de Engenharia Química e de Petróleo \\ E-mail para contato: isabelabarreto@id.uff.br
}

\begin{abstract}
RESUMO - Neste trabalho, com o auxílio do software científico Scilab®, implementaram-se os algoritmos de otimização Nelder Mead Simplex e Newton Raphson, além do modelo cinético utilizado para descrever os processos de pirólise rápida e super-rápida da biomassa de madeira de carvalho. A avaliação das temperaturas ótimas para produção de bio-óleo foi feita por meio do pré-fixamento de valores de tempo inferiores a 10 segundos. Paralelamente, foi possível observar o comportamento das frações mássicas do bio-óleo, sólidos residuais e do produto gasoso em relação à temperatura e/ou ao tempo de reação.
\end{abstract}

\section{INTRODUÇÃO}

Uma das grandes preocupações mundiais, atualmente, está relacionada à produção de energia de modo sustentável, visando-se uma maior conservação do meio ambiente, o que não vem sendo alcançado com o uso dos combustíveis fósseis tradicionais. Diante deste cenário, o número de pesquisas objetivando fontes de energia renováveis tem aumentado, como, por exemplo, a aplicação de biomassas. Dentre os diferentes tipos de biomassas, destacam-se as denominadas de lignocelulósicas, que são compostas, principalmente, por celulose, hemicelulose e lignina, que se encontram presentes nas faixas de, respectivamente, $20-40 \%$, 40-60\%, e 10-25\% em peso, segundo Lima (2017).

Por meio da aplicação dos processos de pirólise rápida e super-rápida, a biomassa é submetida a altas temperaturas em um ambiente com pouco ou nenhum oxigênio, originandose uma fase líquida de alto valor energético denominada de bio-óleo (Sadhukhan et al., 2014). O bio-óleo é uma mistura complexa de substâncias aromáticas, sendo utilizado como combustível, além de ser fonte de vários produtos químicos, como o fenol. Assim como em qualquer outro processo químico, é interessante ter um conhecimento prévio das condições operacionais ótimas visando-se obter o produto de interesse, que neste caso é o bio-óleo. Para tal, é necessária a modelagem matemática do processo, com posterior aplicação de métodos de otimização já difundidos na literatura clássica sobre o tema (Nocedal e Wright, 1999).

\section{METODOLOGIA}

\subsection{Modelagem matemática da pirólise de biomassas}

O modelo utilizado para descrever o processo de pirólise de biomassas é o desenvolvido por Miller e Bellan (1997). Neste, há uma abordagem da biomassa considerando sua 


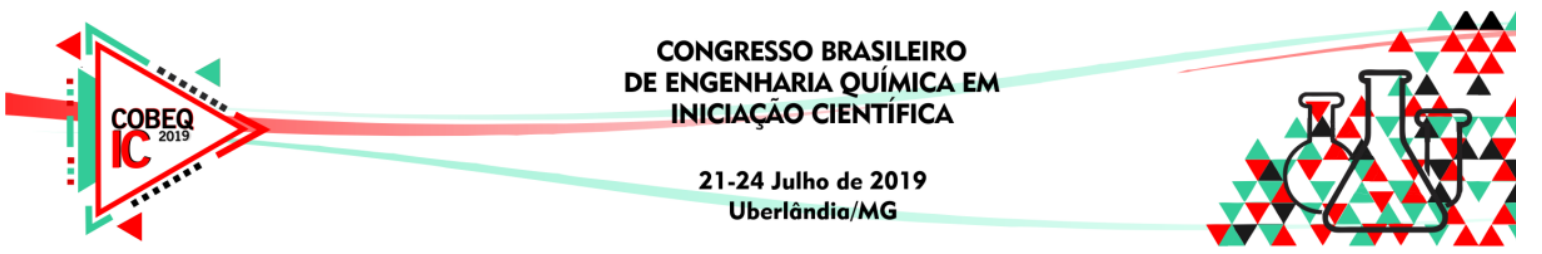

composição como lignina, hemicelulose e celulose, de modo a ignorar possíveis interações que possam ocorrer entre elas. As fases líquida, sólida e gasosa já se encontram determinadas, não havendo necessidade de uma abordagem termodinâmica. Ademais, considera-se que possíveis efeitos referentes aos fenômenos de transporte sejam desprezíveis, levando-se em consideração apenas a cinética das reações empregadas. Os três componentes da biomassa apresentam o mesmo mecanismo de reação, que se encontra indicado na Figura 1.

Figura 1- Esquema genérico de reação do modelo cinético da celulose, hemicelulose e lignina desenvolvido por Miller e Bellan (1997).

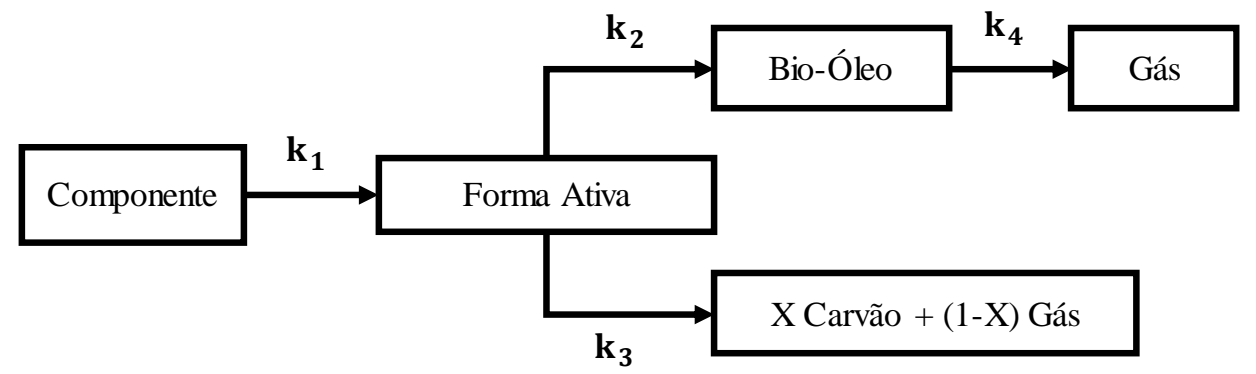

Todas as reações apresentadas na Figura 1 são de primeira ordem e irreversíveis. A reação de inicialização, representada pela constante $\mathrm{k}_{1}$, é uma reação de despolimerização. $\mathrm{O}$ parâmetro $X$ foi obtido experimentalmente, apresentando valores para celulose, hemicelulose e lignina de respectivamente 0,$35 ; 0,60$ e 0,75 . Para o cálculo dos k's, utilizaram-se equações de Arrenhius, cujos fatores pré-exponenciais $(A)$ e as energias de ativação $\left(E_{a}\right)$ das reações em questão se encontram na Tabela 1 . Nota-se que a constante $\mathrm{k}_{4}$, relativa a formação secundária de gás, é a única que independe do tipo de componente. Os sobrescritos $\mathrm{c}$, h e 1 se referem à celulose, hemicelulose e lignina, respectivamente.

Tabela 2 - Parâmetros das constantes de reação por Miller e Bellan (1997).

\begin{tabular}{|c|c|c|}
\hline $\begin{array}{c}\text { Constante de } \\
\text { reaçã̃o }\end{array}$ & $\mathrm{A}\left(\mathrm{s}^{-1}\right)$ & $\mathrm{E}_{\mathrm{a}}\left(\mathrm{KJmol}^{-1}\right)$ \\
\hline \hline $\mathrm{k}_{1}{ }^{\mathrm{c}}$ & $2,8.10^{19}$ & 242,4 \\
\hline $\mathrm{k}_{2}{ }^{\mathrm{c}}$ & $3,28.10^{14}$ & 196,5 \\
\hline $\mathrm{k}_{3}{ }^{\mathrm{c}}$ & $1,3.10^{10}$ & 150,5 \\
\hline $\mathrm{k}_{1}{ }^{\mathrm{h}}$ & $2,1.10^{16}$ & 186,7 \\
\hline $\mathrm{k}_{2}{ }^{\mathrm{h}}$ & $8,75.10^{15}$ & 202,4 \\
\hline $\mathrm{k}_{3}{ }^{\mathrm{h}}$ & $2,6.10^{11}$ & 145,7 \\
\hline $\mathrm{k}_{1}{ }^{1}$ & $9,6.10^{8}$ & 107,6 \\
\hline $\mathrm{k}_{2}{ }^{\mathrm{l}}$ & $1,5 \cdot 10^{9}$ & 143,8 \\
\hline $\mathrm{k}_{3}{ }^{\mathrm{l}}$ & $7,7.10^{6}$ & 111,4 \\
\hline $\mathrm{k}_{4}$ & $4,28.10^{6}$ & 108,0 \\
\hline
\end{tabular}

As frações mássicas de biomassa (incluindo a lignina, celulose, hemicelulose e suas formas ativas), do carvão, bio-óleo e gás formados são representadas, respectivamente, pelas letras $m_{B}, m_{C}$, mo e $m_{G}$, e suas expressões se encontram nas Equações 1 até 4 . Observa-se que todas essas frações mássicas anteriores são dependentes do tempo de residência (t) no reator. $\mathrm{O}$ reator adotado foi do tipo batelada isotérmico, com mistura ideal e volume constante. $\mathrm{O}$ tipo de biomassa utilizado neste trabalho foi uma madeira de carvalho com $35 \%$ de celulose, $40 \%$ de 


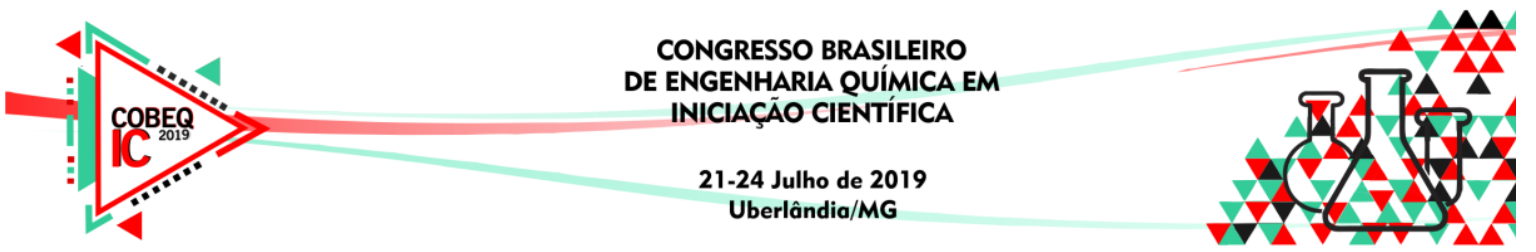

hemicelulose e $25 \%$ de lignina em massa, valores correspondentes aos $m_{i n}$.

$$
\begin{aligned}
& m_{B}(t)=\sum_{j=l, c, h} m_{i n, j} \mathrm{e}^{-k_{1}^{j} t}+\sum_{j=l, c, h} \frac{m_{i n, j} k_{1}^{j}}{k_{2}^{j}+k_{3}^{j}-k_{1}^{j}}\left[\mathrm{e}^{-k_{1}^{j} t}-\mathrm{e}^{-t\left(k_{2}^{j}+k_{3}^{j}\right)}\right] \\
& m_{C}(t)=\sum_{j=l, c, h} \frac{m_{i n, j} k_{1}^{j} k_{3}^{j} X}{k_{2}^{j}+k_{3}^{j}-k_{1}^{j}}\left[\frac{\mathrm{e}^{-k_{1}^{j} t}-1}{k_{1}^{j}}-\frac{\mathrm{e}^{-t\left(k_{2}^{j}+k_{3}^{j}\right)}-1}{k_{2}^{j}+k_{3}^{j}}\right] \\
& m_{O}(t)=\sum_{j=l c h} \frac{m_{i n, j} k_{1}^{j} k_{2}^{j}}{-k_{2}^{j}-k_{3}^{j}+k_{1}^{j}}\left[\frac{\mathrm{e}^{-k_{1}^{j} t}-\mathrm{e}^{-k_{4}^{j} t}}{-k_{4}^{j}+k_{1}^{j}}-\frac{\mathrm{e}^{-t\left(k_{2}^{j}+k_{3}^{j}\right)}-\mathrm{e}^{-k_{4}^{j} t}}{-k_{4}^{j}+k_{2}^{j}+k_{3}^{j}}\right] \\
& m_{G}(t)=\sum_{j=l, c, h}(1-X) k_{3}^{j} \frac{m_{i n, j} k_{1}^{j}}{k_{2}^{j}+k_{3}^{j}-k_{1}^{j}}\left[\frac{\mathrm{e}^{-k_{1}^{j} t}-1}{-k_{1}^{j}}-\frac{\mathrm{e}^{-t\left(k_{2}^{j}+k_{3}^{j}\right)}-1}{-\left(k_{2}^{j}+k_{3}^{j}\right)}\right]+ \\
& +\sum_{j=l, c, h} \frac{m_{i n, j} k_{1}^{j} k_{2}^{j} k_{4}^{j}}{-k_{2}^{j}-k_{3}^{j}+k_{1}^{j}}\left[\begin{array}{l}
\left.\frac{\mathrm{e}^{-k_{1}^{j} t}-1}{-k_{1}^{j}\left(-k_{4}^{j}+k_{1}^{j}\right)}-\frac{\mathrm{e}^{-t\left(k_{2}^{j}+k_{3}^{j}\right)}-1}{-\left(k_{2}^{j}+k_{3}^{j}\right)\left(-k_{4}^{j}+k_{2}^{j}+k_{3}^{j}\right)}+\right] \\
\frac{\mathrm{e}^{-k_{4}^{j} t}-1}{\left(-k_{4}^{j}\right)}\left(\frac{1}{-k_{4}^{j}+k_{2}^{j}+k_{3}^{j}}-\frac{1}{-k_{4}^{j}+k_{1}^{j}}\right)
\end{array}\right]
\end{aligned}
$$

\subsection{Métodos de otimização empregados}

Visando-se maximizar a produção de bio-óleo, optou-se por otimizar os processos de pirólise rápida e super-rápida por meio da minimização do negativo da fração mássica do bioóleo produzido. A temperatura $(\mathrm{T})$ consiste na única variável independente do problema de otimização, sendo o seu valor mínimo de $475 \mathrm{~K}$ de acordo com estudos realizados por Di Blasi e Baeyens (2008). Logo, a função objetivo assume a forma da Equação 5, onde o segundo termo corresponde a uma penalização. Atenta-se que a temperatura é utilizada para o cálculo das constantes de velocidade das reações e, consequentemente, interferindo no valor da fração mássica de bio-óleo. Utilizaram-se tempos de reação fixos ( $\mathrm{t}_{\mathrm{f}}$ ) inferiores a 10 segundos, assim como foram informados chutes iniciais de temperatura ótima de $750 \mathrm{~K}$, sendo esta estimativa um valor coerente segundo Di Blasi e Baeyens (2008).

Para a otimização foram utilizados os algoritmos Nelder Mead Simplex (Nocedal e Wright, 1999) e Newton Raphson (Himmelblau et al, 2001). Ambos os métodos foram desenvolvidos para problemas irrestritos, o que não impossibilita a utilização de termos de penalidade incorporados na função objetivo, como é o caso do presente trabalho. Enquanto o 


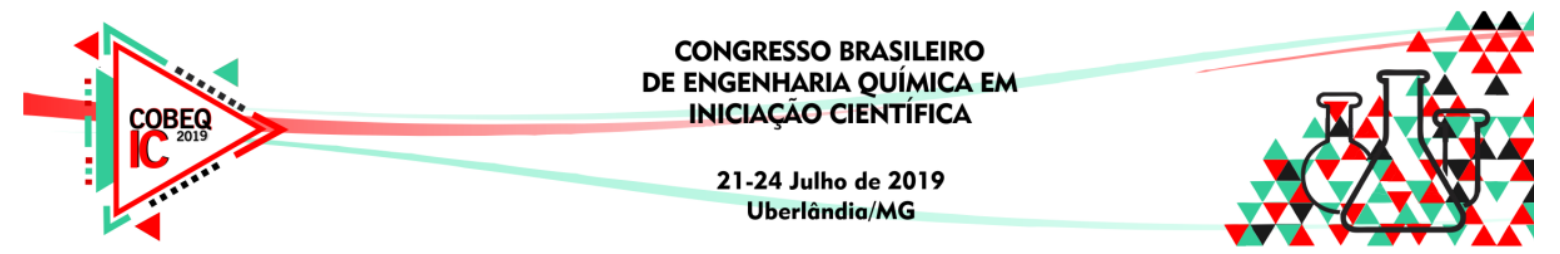

método Simplex consiste em uma busca direta, ou seja, em que se utiliza apenas valores da função objetivo, o método de Newton Raphson utiliza informações de derivadas primeira e segunda, o que o torna mais complexo computacionalmente.

$$
f(T)=-m_{o}(T)+1000 \max (0,475-T)
$$

O algoritmo Nelder Mead Simplex tem sua terminação quando os valores absolutos de $f$ em ambos os vértices do segmento de reta (simplex adotado em caso unidimensional) não diferem mais do que 0,000001. Já no algoritmo de Newton Raphson, o critério de parada consiste em encontrar um valor de temperatura tal que a condição necessária à existência de um extremo irrestrito seja respeitada, com um erro de 0,000001. Essa condição exige que, em um mínimo, a derivada primeira de $f$ com relação a $\mathrm{T}$ seja zero. No Scilab, existe disponível a função "numderivative", que foi utilizada para o cálculo de derivadas por meio de aproximações por diferenças finitas, não necessitando, assim, expressá-las analiticamente.

\section{RESULTADOS E DISCUSSÃO}

Considerando apenas a lignina, obteve-se a variação da fração mássica residual de sólido com o tempo para temperaturas de $673 \mathrm{~K}$ e $873 \mathrm{~K}$, que se encontra na Figura 2. Essa fração mássica corresponde à soma das frações mássicas da lignina, da sua forma ativa, além do carvão originado. Na Figura 2 também se encontra indicado uma comparação com os resultados apresentados por Miller e Bellan (1997), confirmando o sucesso na implementação do modelo. É possível observar que o estado estacionário é alcançado em um menor tempo para a temperatura de $873 \mathrm{~K}$. Nesta situação, a fração mássica de sólido residual é de aproximadamente 0,22, um pouco menos da metade do seu valor em $673 \mathrm{~K}$. Esses resultados podem ser explicados pelo favorecimento da conversão da lignina em maiores temperaturas.

Figura 2- Comparação entre a fração mássica de sólido residual em função do tempo obtida por Miller e Bellan (1997) e pelo atual trabalho para um temperaturas de $673 \mathrm{~K}$ e $873 \mathrm{~K}$.

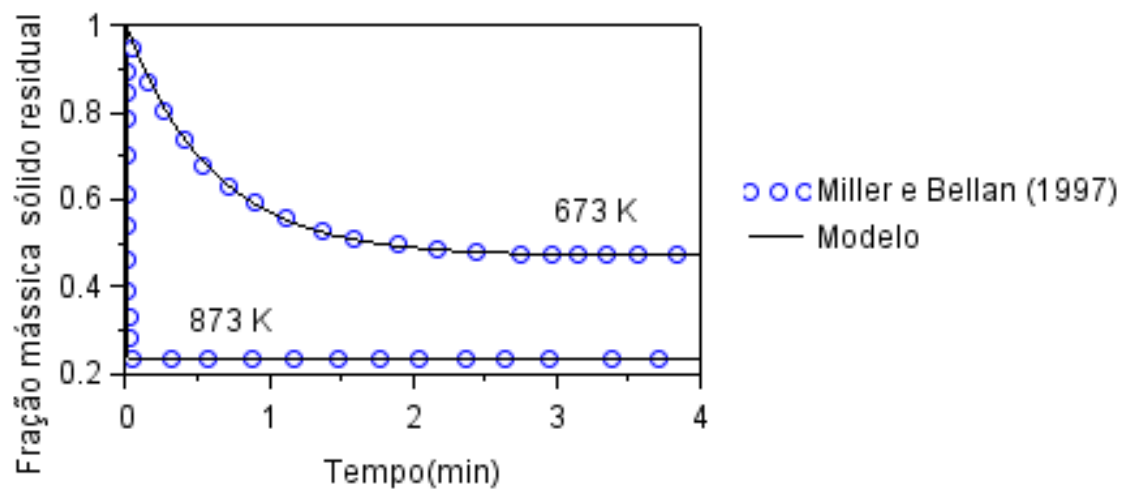

Na Figura 3, encontra-se a aplicação do modelo para a biomassa de madeira de carvalho, ao se considerar a temperatura de reação constante de $750 \mathrm{~K}$. Atenta-se que, neste caso, a fração mássica residual de sólidos também leva em consideração a presença de celulose, hemicelulose e suas respectivas formas ativas. Para a temperatura dada, o bio-óleo apresenta um máximo de produção em aproximadamente 1 segundo e após esse tempo há um decrescimento contínuo, devido favorecimento da ocorrência da reação secundária de conversão do bio-óleo em gás. No 


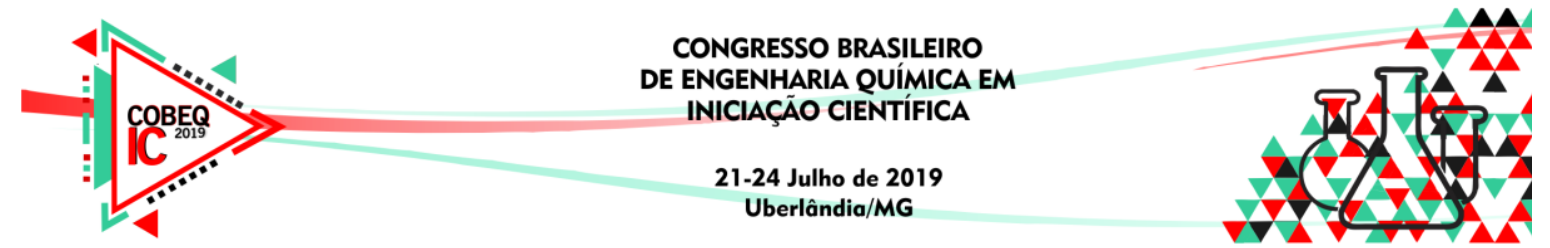

estado estacionário, a fração mássica de sólido residual adquire um valor de aproximadamente 0,15. Para a fase gasosa, a tendência é que haja um crescimento da sua fração mássica até em torno de 0,85 , quando a quantidade de bio-óleo no sistema se torna nula.

Figura 3- Frações mássicas em função do tempo para uma temperatura de $750 \mathrm{~K}$.

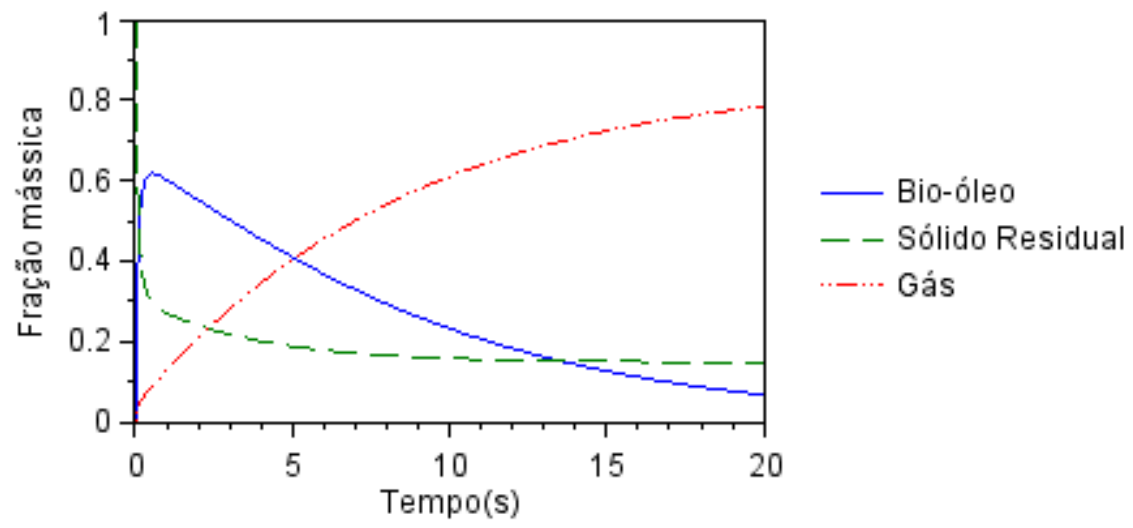

Houve a determinação das temperaturas de operação que maximizam a fração mássica de bio-óleo nos tempos de $0,2 \mathrm{~s} ; 0,4 \mathrm{~s} ; 1 \mathrm{~s} ; 2,5 \mathrm{~s}$ e $5 \mathrm{~s}$. Estes valores são característicos dos processos de pirólise rápida e da super-rápida. Os resultados obtidos para ambos os métodos implementados (Nelder Mead Simplex e Newton Raphson) diferem apenas na terceira casa decimal, sendo por isso considerados idênticos e encontram-se na Tabela 2. Como o valor da maior fração mássica de bio-óleo possível decresce a medida em que o tempo aumenta, ao se trabalhar em condições de pirólise super-rápida obtém-se os melhores resultados. Também é possível observar que, quanto maior o valor do tempo, menor é a temperatura ótima. Ao se utilizar temperaturas inferiores à ótima, tem-se uma região de baixa conversão da biomassa adicionada. Já em temperaturas superiores à ótima há um favorecimento da reação secundária de formação de gás a partir do bio-óleo. Estes resultados podem ser confirmados ao se plotar um gráfico 3D indicado na Figura 4, que permite a visualização do efeito conjunto de temperatura e tempo de reação no rendimento da fração mássica de bio-óleo.

O método de Newton Raphson teve um melhor desempenho, no problema em questão, em relação ao método Simplex, visto que demandou um menor número de iterações. O número de iterações realizadas, em ordem crescente dos valores de tempo, foram de, respectivamente, 5, 6, 4, 4 e 5 para o método de Newton e de, respectivamente 23, 20, 12, 23 e 24 para o método Simplex. Esse comportamento já era previsível, pois um método que leva em consideração a curvatura da função objetivo, por meio do cálculo da derivada segunda, fornece direções de busca mais eficientes. Ambos os métodos demandaram um tempo computacional variando entre milésimos de segundo a 2 segundos, o que é muito satisfatório.

Tabela 2 - Temperaturas ótimas e fração mássica de bio-óleo para diferentes tempos.

\begin{tabular}{|c|c|c|}
\hline Tempo (s) & $\begin{array}{c}\text { Temperatura } \\
\text { ótima }(\mathrm{K})\end{array}$ & $\begin{array}{c}\text { Fração mássica } \\
\text { de bio-óleo }\end{array}$ \\
\hline \hline 0,2 & 813,8 & 0,664 \\
\hline 0,4 & 781,2 & 0,639 \\
\hline 1 & 748,07 & 0,602 \\
\hline 2,5 & 721,20 & 0,557 \\
\hline 5 & 702,76 & 0,518 \\
\hline
\end{tabular}




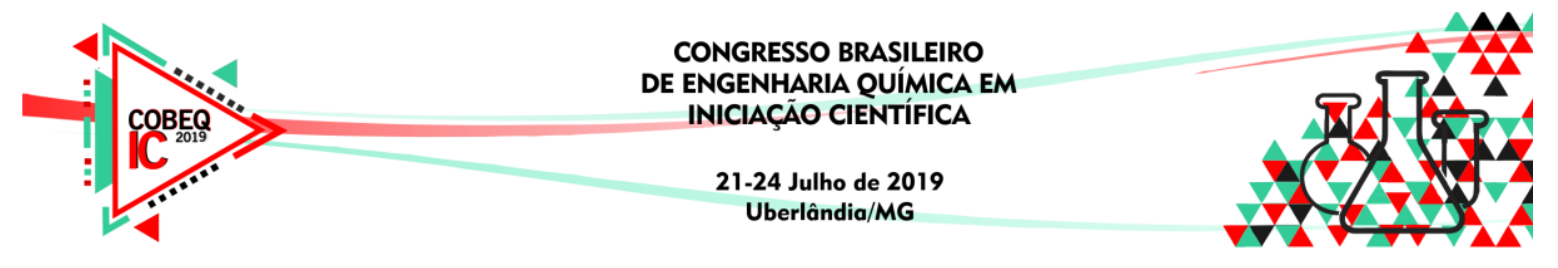

Figura 4-Variação da fração mássica de bio-óleo em função da temperatura e tempo no reator.

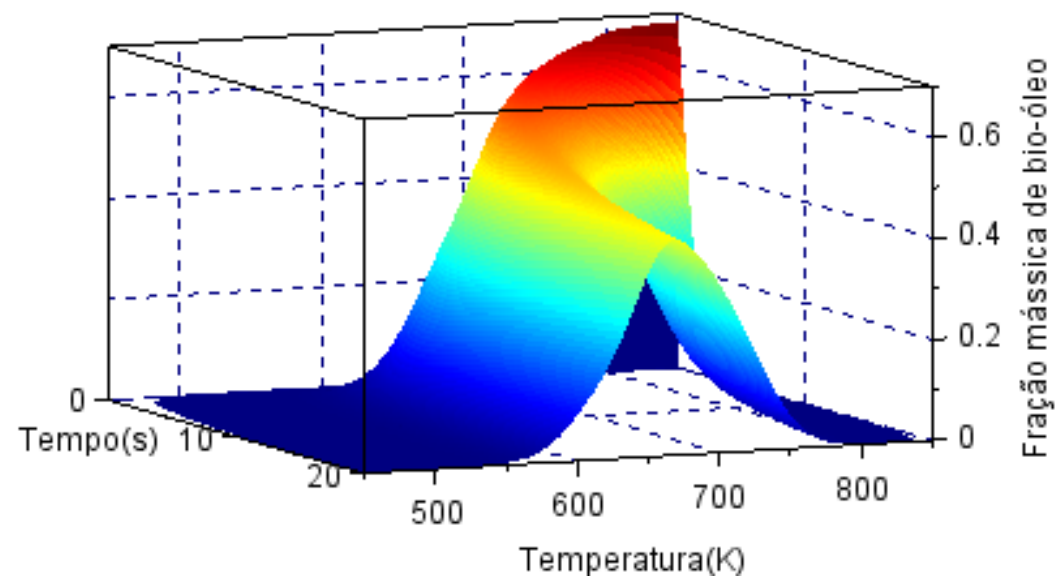

\section{CONCLUSÕES}

O modelo cinético do processo de pirólise de biomassas desenvolvido por Miller e Bellan (1997) foi implementado com sucesso, o que pode ser afirmado por meio da comparação com resultados obtidos pelos próprios autores. Os métodos de otimização Nelder Mead Simplex e Newton Raphson originaram resultados coerentes com o esperado pela literatura, visto que maiores tempo de reação e temperatura favorecem a conversão do bio-óleo em gás. Com isso, explica-se um decrescimento da fração mássica de bio-óleo máxima e da temperatura ótima com o aumento do tempo. Pelo método de Newton Raphson apresentar informações de derivadas ele tende a ter um melhor desempenho em relação ao método Simplex, apesar de ambos os métodos apresentarem ótima performance no presente trabalho.

\section{REFERÊNCIAS}

DI BLASI, C.; BAEYENS, J. Modeling chemical and physical processes of wood and biomass pyrolysis. Progress in Energy and Combustion Science., v. 41, p. 107-121, 2012.

HIMMELBLAU, D. M.; EDGAR, T. F; LASDON, L. Optimization of Chemical Processes. USA: McGraw-Hill, 2001.

LIMA, S.G. Estudo cinético da pirólise de biomassa para produção de combustível limpo. Dissertação (Mestrado) - Departamento de Engenharia Química e de Petróleo, Universidade Federal Fluminense, Niterói, 2017.

MILLER, R. S.; BELLAN, J. A generalized biomass pyrolysis model based on superimposed cellulose, hemicellulose and lignin kinetics. Combustion Science, v. 126, p. 97-137, 1997.

NOCEDAL, J.; WRIGHT, S. J. Numerical Optimization. New York: Springer-Verlag Inc, 1999.

SADHUKHAN, J.; NG, K. S; HERNANDEZ, E, M. Biorefineries and Chemical Processes. Design, Integration and Sustainability Analysis. United Kingdom: John Wiley Sons Ltd, 2014. 